\title{
FULL LENGTH RESEARCH ARTICLE \\ MOTION OF PARTICLES OF NON-ZERO REST MASSES EXTERIOR TO ASTROPHYSICALLY REAL OR HYPOTHETICAL SPHERICAL DISTRIBUTIONS OF MASS WHOSE TENSOR FIELD VARIES WITH POLAR ANGLE ONLY
}

\author{
${ }^{*}$ CHIFU, E. N1; HOWUSU, S. X. K.2; SEYDOU, H. ${ }^{3}$ \& LUMBI, L. W4 \\ ${ }^{1}$ Physics Department, Gombe State University, P.M.B. 127, Gombe, Nigeria \\ ${ }^{2}$ Physics Department, Kogi State University, Anyihba, Kogi State, Nigeria \\ ${ }^{3}$ Physics Department, Gombe State University, P.M.B. 127, Gombe, Nigeria \\ ${ }^{4}$ Physics Department, Nasarawa State University, Keffi, Nigeria.
}

*(Corresponding author)

ebenechifu@yahoo.com

\begin{abstract}
In this article, we extend the metric tensor exterior to astrophysically real or imaginary spherical distributions of mass whose tensor field varies with polar angle only; to derive equations of motion for test particles in this field. The time, radial, polar and azimuthal equations of motion for particles of non-zero rest masses moving in this gravitational field have been derived. The expression for the variation of the time on a clock moving in this gravitational field has been obtained for pure radial motion, the particles move with a constant velocity.
\end{abstract}

Keywords: Tensor field, spherical distribution, mass, motion, polar angle

\section{INTRODUCTION}

The 1907 generalized equivalence hypothesis demands that equations of Physics should be generally covariant, the effect of which physical laws should be valid for any choice of space-time coordinate (Berry 1989). The derivation of tensor equations involves the manipulation of any of the fourteen orthogonal curvilinear coordinates in nature to find solutions to physical problems (Howusu 2007)

In Schwarzschild's well known metric, the tensor field varies only with radial distance (Howosu 2003). This article introduces an astrophysically real or hypothetical distribution of mass within regions of spherical geometry; whose tensor field varies only with polar angle. Although astrophysical bodies having this geometry have not yet been discovered, our analysis in this article will help in studying astrophysical spherical distributions of mass whose field varies with both radial distance and polar angle only. An example of such a distribution is; a homogenous distribution of mass within a spherical region, which is rotating with uniform angular speed about a fixed diameter (Howosu 2003, 2007).

\section{Theoretical Analysis}

Consider a spherical body of radius $R$ and total rest mass $M$ distributed uniformly with a density $\rho$. Then the general relativistic field equation in its exterior region are given tensorially (Howosu 2007).

$$
G_{\mu v}=0
$$

where $G_{\mu v}$ is the Einstein tensor. As in usual notation, the Greek subscripts run from 0 to 3 , with the $0^{\text {th }}$ component representing the time coordinate, and the $1^{\text {st }}, 2^{\text {nd }}$ and $3^{\text {rd }}$ components denote the location in space.

Schwarzschild's metric is the solution of Einstein's gravitational field equation exterior to a static homogenous spherical body (Peter \& Dunsby 2000; Howosu 2003; 2007) given by:

$$
\begin{aligned}
& g_{00}=1+\frac{2}{c^{2}} f(r) \\
& g_{11}=-\left[1+\frac{2}{c^{2}} \mathrm{f}(\mathrm{r})\right]^{-1} \\
& g_{22}=-r^{2} \\
& g_{33}=-r^{2} \sin ^{2} \theta \\
& g_{\mu v}=0 ; \quad \text { otherwise }
\end{aligned}
$$

where $c$ is the speed of light in vacuum. $f(r)$ is an arbitrary function determined by the distribution; it is a function of the radial coordinate $r$ only since the distribution and hence its exterior gravitational field possess spherical symmetry. From the condition that these metric components should reduce to the field of a point mass located at the origin (Howosu 2007) and contain Newton's equations of motion in the gravitational field of the static homogenous spherical body, it follows that $f(r)$ is the Newtonian gravitational scalar potential in the exterior region of the body, defined in this field as

$f(r)=-\frac{G M}{r}$

where $G$ is the universal gravitational constant.

Now, let us consider a mass distribution within spherical geometry in which the tensor field varies with polar angle only. The covariant metric tensor for the gravitational field exterior to this distribution of mass or pressure is given as (Howosu 2007).

$$
\begin{aligned}
& g_{00}=1+\frac{2}{c^{2}} f(\theta) \\
& g_{11}=-\left[1+\frac{2}{c^{2}} \mathrm{f}(\theta)\right]^{-1}
\end{aligned}
$$


$g_{22}=-r^{2}$

$g_{33}=-r^{2} \sin ^{2} \theta$

$g_{\mu v}=0 ; \quad$ otherwise

where $f(\theta)$ is an arbitrary function determined by the astrophysical distribution and posseses all the symmetries of the latter a priori.

To obtain the corresponding contravariant metric tensor for this gravitational field, we apply the Quotient Theorem (Arfken 1995) of tensor analysis:

$g^{\mu \sigma} \cdot g_{\sigma v}=\delta_{v}^{\mu}$

where $\delta_{v}^{\mu}$ is the Kronecker delta tensor defined by

$\delta_{v}^{\mu}=\left\{\begin{array}{l}0, \mu \neq v \\ 1, \mu=v\end{array}\right.$

By imposing the Quotient Theorem of tensor analysis (equation 13) on the covariant metric tensor yields the components of the contravariant tensor as

$$
\begin{aligned}
& g^{00}=\left[1+\frac{2}{c^{2}} f(\theta)\right]^{-1} \\
& g^{11}=-\left[1+\frac{2}{c^{2}} f(\theta)\right] \\
& g^{22}=-r^{-2} \\
& g_{33}=-\left(r^{2} \sin ^{2} \theta\right)^{-1} \\
& g^{\mu \nu}=0 ; \text { otherwise }
\end{aligned}
$$

The coefficients of affine connection, defined by the metric tensor of space-time are found to be given in terms of the metric tensor as

$\Gamma_{02}^{0}=\Gamma_{20}^{0}=\frac{1}{2} g^{00} g_{00.2}$

$\Gamma_{12}^{1}=\Gamma_{21}^{1}=\frac{1}{2} g^{11} g_{11,2}$

$\Gamma_{22}^{1}=-\frac{1}{2} g^{11} g_{22,1}$

$\Gamma_{33}^{1}=-\frac{1}{2} g^{11} g_{33,1}$

$\Gamma_{00}^{2}=-\frac{1}{2} g^{22} g_{00,2}$

$\Gamma_{11}^{2}=-\frac{1}{2} g^{22} g_{11,2}$

$\Gamma_{12}^{2}=\Gamma_{21}^{2}=\frac{1}{2} g^{22} g_{22,1}$

$$
\begin{aligned}
& \Gamma_{33}^{2}=-\frac{1}{2} g^{22} g_{33,2} \\
& \Gamma_{13}^{3}=\Gamma_{31}^{3}=\frac{1}{2} g^{33} g_{33,1} \\
& \Gamma_{23}^{3}=\Gamma_{32}^{3}=\frac{1}{2} g^{33} g_{33,2} \\
& \Gamma_{\beta \gamma}^{\alpha}=0 ; \quad \text { otherwise }
\end{aligned}
$$

where the comma denotes partial differentiation with respect to $(0,1,2)$ $=(c t, r, \theta)$. Equations (20) to (30) can be written more explicitly in terms of $(c t, r, \theta, \phi)$ as

$$
\begin{aligned}
& \Gamma_{02}^{0}=\Gamma_{20}^{0}=\frac{1}{c^{2}}\left[1+\frac{2}{c^{2}} \mathrm{f}(\theta)\right]^{-1} \frac{d f(\theta)}{d \theta} \\
& \Gamma_{12}^{1}=\Gamma_{21}^{1}=-\frac{1}{c^{2}}\left[1+\frac{2}{\mathrm{c}^{2}} \mathrm{f}(\theta)\right]^{-1} \frac{d f(\theta)}{d \theta} \\
& \Gamma_{22}^{1}=-\mathrm{r}\left[1+\frac{2}{\mathrm{c}^{2}} \mathrm{f}(\theta)\right] \\
& \Gamma_{33}^{1}=-\mathrm{r} \sin \theta\left[1+\frac{2}{\mathrm{c}^{2}} \mathrm{f}(\theta)\right] \\
& \Gamma_{00}^{2}=\frac{1}{r^{2} c^{2}} \frac{d f}{d \theta} \\
& \Gamma_{11}^{2}=\frac{1}{r^{2} c^{2}}\left[1+\frac{2}{c^{2}} f(\theta)\right]^{-2} \frac{d f}{d \theta} \\
& \Gamma_{12}^{2}=\Gamma_{21}^{2}=\mathrm{r}-1 \\
& \Gamma_{33}^{2}=-\frac{1}{2} \sin 2 \theta \\
& \Gamma_{13}^{3}=\Gamma_{31}^{3}=\mathrm{r}-1 \\
& \Gamma_{23}^{3}=\Gamma_{32}^{3}=\cot \theta \\
& \Gamma_{\beta \gamma}^{\alpha}=0 ; \\
& \text { Otherwise }
\end{aligned}
$$

It is well known that the general relativistic equation of motion for particles of non-zero rest masses in any gravitational field are given by (Bergmann 1987).

$\frac{d^{2} x^{\mu}}{d \tau^{2}}+\Gamma_{v \lambda}^{\mu}\left(\frac{d x^{\nu}}{d \tau}\right)\left(\frac{d x^{\lambda}}{d \tau}\right)=0$

where $\tau$ is the proper time. Setting $\mu=0$ in equation (42) gives the time equation of motion as

$\frac{d^{2} x^{0}}{d \tau^{2}}+\Gamma_{v \lambda}^{0}\left(\frac{d x^{v}}{d \tau}\right)\left(\frac{d x^{\lambda}}{d \tau}\right)=0$

Substituting equation (31 into (43) gives

$\frac{d^{2} x^{0}}{d \tau^{2}}+2 \Gamma_{02}^{0} \frac{d x^{0}}{d \tau} \frac{d x^{2}}{d \tau}=0$

Fonıation (12) r.an he written more exnlicitly as 
$\ddot{t}+\frac{2}{c^{2}}\left[1+\frac{2}{c^{2}} f(\theta)\right]^{-1} \frac{d f(\theta)}{d \theta} \dot{t} \dot{\theta}=0$

where the dot denotes differentiation with respect to proper time, $\tau$. Equation (45) is the time equation of motion for particles of non-zero rest masses in this gravitational field. It is instructive and interesting to note that equation (45) can equally be written as

$$
\begin{aligned}
& \frac{\ddot{t}}{\dot{t}}+\frac{2 \frac{d f}{d \theta}}{c^{2}\left[1+\frac{2}{c^{2}} f(\theta)\right]} \dot{\theta}=0 \\
& \frac{d}{d \tau}(\ln \dot{t})+\frac{d}{d \tau} \ln \left[1+\frac{2}{c^{2}} f(\theta)\right]=0
\end{aligned}
$$

Thus,

$$
\dot{t}=A\left[1+\frac{2}{c^{2}} f(\theta)\right]^{-1}
$$

where $A$ is a constant of motion. But, as $t \rightarrow \tau, f(\theta) \rightarrow 0$ and thus $A=1$. Equation (48) thus becomes

$$
\dot{t}=\left[1+\frac{2}{c^{2}} f(\theta)\right]^{-1}
$$

Equation (49) is the expression for the variation of the time on a clock with this gravitational field. Similarly, setting $\mu=1$ in equation (42) gives the radial equation of motion as

$$
\ddot{r}-\frac{2}{c^{2}}\left[1+\frac{2}{c^{2}} f(\theta)\right]^{-1} \frac{d f(\theta)}{d \theta} \dot{r} \dot{\theta}-r\left[1+\frac{2}{c^{2}} f(\theta)\right] \dot{\theta}^{2}-r \sin ^{2} \theta\left[1+\frac{2}{c^{2}} f(\theta)\right] \dot{\phi}^{2}=0
$$

For pure radial motion $\dot{\theta}=\dot{\phi} \equiv 0$ and hence the radial equation (18) becomes

$$
\ddot{r}=0
$$

Equation (51) is the pure radial equation of motion for particles of nonzero rest masses in this field. Thus, the instantaneous pure radial speed of a particle of non-zero rest mass in this gravitational field is constant.

Also, setting $\mu=2$ and $\mu=3$ in equation (51) gives the respective polar and azimuthal equations of motion for particles of non-zero rest masses in this field as

$\ddot{\theta}+\frac{1}{r^{2}} \frac{d f(\theta)}{d \theta} \dot{t}^{2}+\frac{1}{r^{2} c^{2}}\left[1+\frac{2}{c^{2}} f(\theta)\right]^{-2} \frac{d f(\theta)}{d \theta} \dot{r}^{2}-\frac{1}{2} \sin 2 \theta(\dot{\phi})^{2}+\frac{2}{r} \dot{r} \dot{\theta}=0$

and

$$
\ddot{\phi}+\frac{2}{r} \dot{r} \dot{\phi}+2 \cot \theta(\dot{\theta})(\dot{\phi})=0
$$

It is instructive to note that equation (53) is equal to the azimuthal equation of motion for particles of non-zero rest masses in Schwarzschild's field (Bergmann 1987; Peter \& Dunsby 2000; Howosu 2003; Howosu 2007. Thus, the instantaneous azimuthal angular velocity from our field is exactly the same as that obtained from Newton's theory of gravitation (Howosu 2005) and Schwarzschild's metric.

\section{Remarks and Conclusion}

The time, radial, polar and azimuthal equations of motion for particles of non-zero rest masses exterior to astrophysically real or hypothetical spherical distribution of mass, whose tensor field varies only with polar angle were found to be equations (45), (50), (52) and (53) respectively. The immediate consequences of the results obtained in this paper are

The solution to the time equation of motion gives the variation of the time on a clock with the gravitational field. Thus, the expression for gravitational time dilation in this gravitational field has been obtained.

The radial equation of motion can be used to obtain the instantaneous speed of a particle of non-zero rest mass in this field. For pure radial motion, the instantaneous speed of a particle of non-zero rest mass is found to be constant

The azimuthal equation of motion is the same as that obtained from Schwarzschild's field and Newton's Theory of gravitation. Hence, our field has no effect on the azimuthal motion of particles.

The coefficients of affine connection obtained can equally be used to construct the Riemann-Christoffel, Ricci and Einstein's tensor for this field and hence the Einstein field equations for this gravitational field can be obtained.

The resultant Einstein field equations obtained from (iv) contain only a single unknown $f(\theta)$ and thus they can be solved to obtain explicit values for $f(\theta)$.

\section{REFERENCES}

Arfken, G. 1995. Mathematical Methods for Physicists, $5^{\text {th }}$ edition, Academic Press, New York

Bergmann, P. G. 1987. Introduction to the Theory of Relativity, Prentice Hall, India, 203-ff

Berry, M. V. 1989. Principles of Cosmology and Gravitation. IPO Publishing Ltd, London

Dunsby, P. K. S. 2000. An Introduction to Tensors and Relativity Cape Town, 51-110 http://shiva.mth.uct.ac.za/gr96/gr96.html

Howusu, S. X. K. 2003. Discourse on General Relativity. Jos University Press Ltd

Howusu, S. X. K. Musongong, E. F. 2005. Newton's Equations of Motion in the Gravitational Field of an Oblate Mass, Galilean Electrodynamics 16(5)

Howusu, S. X. K. 2007. The 210 Astrophysical Solutions plus 210 Cosmological Solutions of Einstein's Geometrical Gravitational Field Equations. Natural Philosophy Society Jos, 2-227 